\title{
Computational prediction of miRNA genes from small RNA sequencing data
}

\section{Wenjing Kang and Marc R. Friedländer*}

Science for Life Laboratory, Department of Molecular Biosciences, The Wenner-Gren Institute, Stockholm University, Stockholm, Sweden

Edited by:

Alessandro Laganà, The Ohio State University, USA

\section{Reviewed by:}

Noam Shomron, Tel Aviv University, Israel

Patrick Xuechun Zhao, Samuel

Roberts Noble Foundation, USA

\section{*Correspondence:}

Marc R. Friedländer, Science for Life Laboratory, Department of Molecular Biosciences, The Wenner-Gren Institute, Stockholm University, Box 1031, Solna 17121, Sweden e-mail:marc.friedlander@scilifelab.se
Next-generation sequencing now for the first time allows researchers to gage the depth and variation of entire transcriptomes. However, now as rare transcripts can be detected that are present in cells at single copies, more advanced computational tools are needed to accurately annotate and profile them. microRNAs (miRNAs) are 22 nucleotide small RNAs (sRNAs) that post-transcriptionally reduce the output of protein coding genes. They have established roles in numerous biological processes, including cancers and other diseases. During miRNA biogenesis, the sRNAs are sequentially cleaved from precursor molecules that have a characteristic hairpin RNA structure. The vast majority of new miRNA genes that are discovered are mined from small RNA sequencing (sRNA-seq), which can detect more than a billion RNAs in a single run. However, given that many of the detected RNAs are degradation products from all types of transcripts, the accurate identification of miRNAs remain a non-trivial computational problem. Here, we review the tools available to predict animal miRNAs from sRNA sequencing data. We present tools for generalist and specialist use cases, including prediction from massively pooled data or in species without reference genome. We also present wet-lab methods used to validate predicted miRNAs, and approaches to computationally benchmark prediction accuracy. For each tool, we reference validation experiments and benchmarking efforts. Last, we discuss the future of the field.

Keywords: miRNA, microRNA, gene prediction, next-generation sequencing data

\section{MIRNA BIOLOGY}

microRNAs (miRNAs) are a class of small RNAs (sRNAs) around 22 nucleotides in length. They are never translated, but posttranscriptionally reduce the output of protein coding genes (Kloosterman and Plasterk, 2006; Bushati and Cohen, 2007; Farazi et al., 2008; Ghildiyal and Zamore, 2009). They have been found in all animals studied, in numbers that appear to correlate with organismal complexity, for instance, nematodes have around 200 miRNA genes while humans have more than 3000 (Kozomara and Griffiths-Jones, 2011; Friedländer et al., 2014). Mutant animals that are void of miRNAs either die at early embryonic stages or have severe developmental defects, showing the importance of the regulation they infer (Bernstein et al., 2003; Giraldez et al., 2005; Morita et al., 2007; Wang et al., 2007). More than half of all protein coding transcripts are estimated to be under regulation of miRNAs in one or more cellular contexts (Friedman et al., 2009). Thus, it is not surprising that miRNAs are involved in numerous biological contexts, ranging from formation of cell identify to development (Stefani and Slack, 2008).

\section{miRNA BIOGENESIS}

The majority of miRNAs are transcribed by Polymerase II and have features similar to protein coding transcripts: a $5^{\prime}$ cap, exons, and a poly(A)-tail (Figure 1). Each of the primary transcripts harbors one or more characteristic RNA hairpin structures around 60 nucleotides in length. While in the nucleus, these structures can be recognized by the Microprocessor complex, consisting of Drosha and DGCR8 proteins, which cleave the hairpin out of the primary transcript (Denli et al., 2004; Gregory et al., 2004; Han et al., 2004; Landthaler et al., 2004). The hairpin is then exported to the cytosol, where it undergoes a second cleavage by Dicer, a canonical component of the RNA interference pathway (Bernstein et al., 2001; Hutvagner et al., 2001; Ketting et al., 2001; Knight and Bass, 2001). The cleavage releases three products: the mature miRNA guide strand, the miRNA passenger strand, and the loop. These three products fall in determined positions: the guide and the passenger form an RNA duplex with two nucleotides $3^{\prime}$ overhangs, while the loop consists of the terminal end of the hairpin, positioned between the guide and the passenger strands (Ha and Kim, 2014). While the loop and the passenger strands are generally degraded as bi-products of the biogenesis, the guide miRNA remains bound to an Argonaute protein, which is part of the miRNP complex. It is not always the same strand that is fated to be bound to the Argonaute protein, in the case of many miRNA hairpins either strand can be incorporated and repress targets (Okamura et al., 2008; Guo and Lu, 2010; Yang et al., 2011). The mature miRNA can guide the effector complex to target sites, typically located in $3^{\prime}$ UTRs of mRNAs, through partial base complementarity (Lai, 2002; Bartel, 2009). Once bound, the complex reduces protein output of the transcript, either by destabilizing it through shortening of the poly-A tail, inhibiting its translation or by re-localizing it to subcellular ribo-protein particles, where it is inaccessible to the translation machinery (Filipowicz et al., 2008; Huntzinger and Izaurralde, 2011). Some miRNAs follow non-canonical biogenesis 


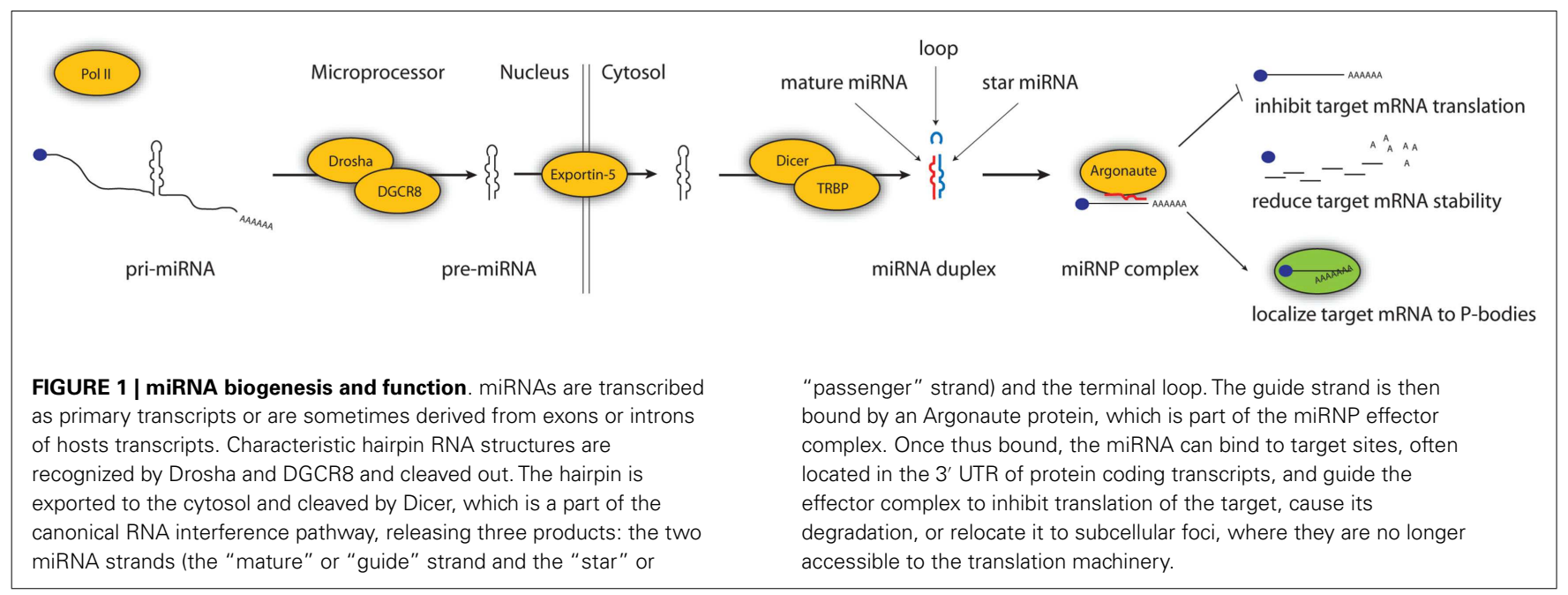

pathways, but are believed to function like the canonical sequences (Ha and Kim, 2014). Altogether, it is estimated that around 60\% of all human protein coding transcripts are regulated by miRNAs in one or more cellular conditions (Friedman et al., 2009).

\section{miRNAs IN HUMAN DISEASE}

Given the prevalence of miRNA regulation, it is not surprising that miRNAs have been involved in numerous human diseases. These regulators appear to play particularly critical roles in cancers, where they can function as onco-genes or tumor suppressors. For instance, the miR-17-92 cluster is found to be up-regulated in several cancers (He et al., 2005), and miR-15 and miR-16 are often deleted in leukemias (Cimmino et al., 2005). Although some miRNAs can function as onco-genes, they are in most cases downregulated individually or collectively in cancers (Medina and Slack, 2008). miRNAs are important in cell differentiation and formation of cell identity, and often cancer cells revert to more undifferentiated states. In addition to cancers, miRNAs have been involved in many types of diseases including: cardiovascular, immunological, neurodegenerative, and psychiatric (Taft et al., 2010; Esteller, 2011). In disease, miRNA function can be perturbed in several ways: by down-regulation of the biogenesis factors (Hill et al., 2009), by mutation in the miRNA locus (Mencia et al., 2009), by loss or gains of the miRNA genes (Zhang et al., 2006b), or by epigenetic changes such as hypermethylation (Davalos et al., 2012). There are also cases where disease is caused by mutations that destroy (Christensen et al., 2009) or create (Abelson et al., 2005) target sites in the $3^{\prime}$ UTR of protein coding transcripts.

Before the role of a miRNA in a given disease can be investigated, it must be discovered and annotated. Many miRNAs have specific expression patterns and may not be highly expressed outside the particular tissue that is studied, and may not yet have been discovered. Therefore, miRNA prediction is an important first analysis step of sRNA-seq analysis in clinical context. miRNA prediction can also be used for basic research, when annotating the complement of regulatory RNAs in emerging model systems. The purpose of this review is to present the methods used to discover new animal or human miRNA genes from sRNA-seq data. We will focus on published methods that can be downloaded and run, without the user needing to implement algorithms as software by him/herself. We will discuss the strengths of the distinct methods, and will reference the studies in which the methods have been benchmarked computationally. Thus, this review can serve as a platform for the reader to decide which method is ideally suited for his miRNA prediction use case. Finally, we will present low and high-throughput methods to validate the discovered miRNA candidates.

\section{MIRNA PREDICTION PREDICTION FROM GENOME SEOUENCE}

The biogenesis of miRNAs is key to their discovery. When the field was still young and little data were available, researchers would search the genome sequences for loci that would give rise to RNA hairpin structure if transcribed. These methods have combined structure prediction with either scoring (Lai et al., 2003; Lim et al., 2003; Ohler et al., 2004; Wang et al., 2005) or rules-based (Dezulian et al., 2006; Zhang et al., 2006a) or machine-learning classification (Nam et al., 2005; Jiang et al., 2007; Sheng et al., 2007) of the hairpin features. Some of the methods have incorporated conservation information into the prediction; in fact, one approach has used phylogenetic shadowing to detect the characteristic conservation profile of miRNAs, where the miRNA strands are more conserved in sequence than the terminal loop (Berezikov et al., 2005). However, it is impossible to know from the genome DNA sequence if a locus is really transcribed and gives rise to mature miRNAs. Thus, considering the size of most animal genomes, these methods yield many false positive hairpins that are either not transcribed or do not interact with the biogenesis factors. For instance, in the human genome, around 11 million loci would give rise to hairpin structures if transcribed (Bentwich, 2005), but only a few thousands of them are actually cleaved to mature miRNAs (Kozomara and Griffiths-Jones, 2011; Friedländer et al., 2014).

\section{SANGER SEQUENCING}

For an unbiased detection of miRNAs, methods were developed to directly sequence sRNAs. This was done by separating them from other transcripts on high-resolution gels, and sequencing by Sanger sequencing (Lagos-Quintana et al., 2001; Lau et al., 
2001; Lee and Ambros, 2001). Because of the limited throughput of this technology, typically just a few hundreds of sRNAs were detected, and many of these would be degradation products of longer transcripts such as mRNAs, rRNAs, and tRNAs, or even from un-annotated transcripts. To ensure that the predicted miRNAs were genuine, researchers would filter out sequences mapping to known non-miRNA transcript annotations, and would require that the predicted miRNA was located in a loci that could give rise to a hairpin transcript (Ambros et al., 2003). More specifically and in accordance with miRNA biogenesis, the predicted sequence should be located on a hairpin arm. Further, if two sequences should locate to the same hairpin, it was required that they should form a duplex with two nucleotide $3^{\prime}$ overhangs, as expected from Dicer processing.

\section{NEXT-GENERATION SEQUENCING}

In 2006, the first next-generation sequencing instruments became commercially available, allowing orders of magnitude increase in data generation. For instance, the current Illumina HiSeq 2500 instruments can sequence around one billion sRNAs in $<2$ days. This sequencing power can be distributed between several experiments, but still sRNA-seq studies detect millions of transcripts per sample. Since a mammalian cell typically contains on the order of 100,000 miRNA transcripts (Calabrese et al., 2007), this means that sequences that are present in less than one molecule per cell can still be detected. This also holds for other clades, for instance, the $l s y-6$ miRNA, which is expressed in only a single neuron in the entire nematode body (Johnston and Hobert, 2003), is now routinely detected in sRNA-seq experiments (unpublished results).

The sensitivity of these sequencing methods means that very lowly expressed sRNAs other than miRNAs are also detected. These can include short interfering RNAs (siRNAs) and piwiinteracting RNAs (piRNAs) but can also be rare degradation products of longer transcripts like rRNAs, tRNAs, and mRNAs or unannotated transcripts. In addition to this, there is now emerging evidence that transcripts like tRNAs can undergo endonucleolytic cleavage at specific positions to produce functional sRNAs (Chen and Heard, 2013). Altogether, this means that sRNAs sequenced in a single experiment can originate from millions of distinct loci in the human genome (Friedländer et al., 2008). The methods that were developed to predict miRNAs from Sanger sequencing should only handle a few thousand loci. Therefore, they are not specific enough to be applied to next-generation sequencing data, and produce numerous false positives. These false positives are transcribed and form hairpins, but the sRNAs generated from them are degradation products resulting from normal RNA turnover. Thus, accurately identifying the miRNAs in this complex landscape of sRNAs is a daunting task.

To reduce false positives, methods to predict miRNAs from sRNA-seq employ post-filtering steps beyond what is used for Sanger sequencing. The next-generation discovery methods almost all require the presence of a hairpin structure, and the formation of a duplex if both miRNA strands are detected. In addition, many methods require that the candidate precursors do not overlap known non-miRNA annotations (Berninger et al., 2008). Hairpins that pass these requirements are then exposed to a further filter step. These steps can be rule-based or can involve probabilistic scoring or machine learning (see below). The features that are evaluated can be divided into structure features and signature features (Friedländer et al., 2008). The first reflect how well the hairpin structure conforms to known miRNA precursors. For instance, most of the nucleotides in the putative duplex should be base paired, and the hairpin should not contain large bulges besides the terminal loop. Some methods also require that the structure should be energetically stable, as this is a hallmark of genuine miRNA hairpins. The signature is a measure of how well the distribution of sequenced RNAs fit in the hairpin structure. For instance, every sequenced RNA should correspond to either guide or passenger strand, or to the terminal loop. The guide and passenger RNAs should form a duplex with two nucleotide $3^{\prime}$ overhangs, as is typical of Dicer processing. Further, it is expected that the candidate miRNA guide strand is detected several times, given the sensitivity of next-generation sequencing. Last, since it is known that processing of Drosha and Dicer produces clearly defined $5^{\prime}$ ends, the sequenced RNAs should align neatly in this end (Ruby et al., 2006).

Besides the core prediction methods, source for predicting miRNAs differ in other respects. This includes the mapping tool, whether read pre-processing is provided, whether the tool has a graphic user interface or must be operated on the command line and whether additional analyses like expression analyses and target predictions are supported. Also, some methods are not just applicable for animal miRNAs, but also for plant sequences. Finally, some methods have been tested by computational benchmarking in several studies and their predictions validated in the wet-lab. In the following section, we describe the tools of the field in alphabetical order (Table 1).

\section{SPECIFIC ALGORITHMS deepBlockAlign}

deepBlockAlign is innovative in that it provides advanced scoring of the read signature, but does not evaluate the RNA structure (Langenberger et al., 2012; Pundhir and Gorodkin, 2013). deepBlockAlign uses a variant of Needleman-Wunsch to identify blocks of mapped reads that have similar features, including read begin positions and block height. In a second step, similar groups of blocks are identified using a variant of the Sankoff algorithm. These groups of blocks correspond to gene loci. To predict novel miRNAs, the method finds loci that have block features similar to known miRNAs. While the profiles might be different for plants and animals, or specific to particular tissues or pathological conditions, the method can compare to all known profiles from the entire miRBase database of miRNAs, giving it good coverage. Since this method does not evaluate the RNA structure, it can predict miRNAs that do not have canonical structure, or whose conformation is not easily predicted by computational methods. Alternatively, it can be combined with down-stream structure analysis, to further improve specificity ${ }^{1}$.

\section{miRanalyzer}

miRanalyzer first removes reads that map to known miRNAs or other transcripts (Hackenberg et al., 2009). The remaining reads

\footnotetext{
${ }^{1}$ http://rth.dk/resources/dba/
} 
Table 1 | Tools for predicting animal miRNAs from sRNA-seq data

\begin{tabular}{|c|c|c|c|c|c|c|c|c|c|}
\hline Tool & Algorithm & $\begin{array}{l}\text { Mapping } \\
\text { tool }\end{array}$ & $\begin{array}{l}\text { Tested in } \\
\text { plants }\end{array}$ & Performance comparison & Validated in wet-lab & $\begin{array}{l}\text { Pre-process } \\
\text { data }\end{array}$ & $\begin{array}{l}\text { Quantifies } \\
\text { expression }\end{array}$ & $\begin{array}{l}\text { Target } \\
\text { prediction }\end{array}$ & $\begin{array}{l}\text { User } \\
\text { interface }\end{array}$ \\
\hline \multicolumn{10}{|c|}{ GENERALTOOLS } \\
\hline deepBlockAlign & $\begin{array}{l}\text { Read block } \\
\text { alignment }\end{array}$ & Not included & Yes & $\begin{array}{l}\text { Langenberger et al. (2012), and } \\
\text { Pundhir and Gorodkin (2013) }\end{array}$ & No & No & No & No & $\begin{array}{l}\text { Graphics, } \\
\text { webserver }\end{array}$ \\
\hline miRanalyzer & Random forest & Prefix tree & No & Hackenberg et al. (2009) & See below & Partial & $\begin{array}{l}\text { Differential } \\
\text { expression }\end{array}$ & $\begin{array}{l}\text { MiRanda and } \\
\text { TargetScan }\end{array}$ & $\begin{array}{l}\text { Graphics, } \\
\text { webserver }\end{array}$ \\
\hline $\begin{array}{l}\text { miRanalyzer } \\
\text { (update) }\end{array}$ & Random forest & Bowtie & Yes & $\begin{array}{l}\text { An et al. (2013), Friedländer et al. } \\
\text { (2012), Hackenberg et al. (2011) } \\
\text { Hansen et al. (2014), Pundhir and } \\
\text { Gorodkin (2013), and Williamson } \\
\text { et al. (2013) }\end{array}$ & $\begin{array}{l}\text { RT-PCR (Smith et al., 2013), } \\
\text { Northern blot (Mayoral et al., } \\
\text { 2014) }\end{array}$ & Yes & $\begin{array}{l}\text { Differential } \\
\text { expression }\end{array}$ & TargetSpy & $\begin{array}{l}\text { Graphics, } \\
\text { webserver, } \\
\text { and } \\
\text { standalone }\end{array}$ \\
\hline miRCat & Rules-based & PatMaN & Yes & Moxon et al. (2008) & $\begin{array}{l}\text { RT-PCR (Kohli et al., 2014, and } \\
\text { Pandey et al., 2014), Northern } \\
\text { blot (Donaszi-Ivanov et al., } \\
\text { 2013) }\end{array}$ & Yes & $\begin{array}{l}\text { Yes (mirprof), } \\
\text { differential } \\
\text { expression } \\
\text { (colide) }\end{array}$ & PAREsnip & $\begin{array}{l}\text { Graphics, } \\
\text { webserver, } \\
\text { and } \\
\text { standalone }\end{array}$ \\
\hline miRDeep & Bayesian & Megablast & No & $\begin{array}{l}\text { An et al. (2013), Friedländer et al. } \\
\text { (2008, 2012), Hendrix et al. (2010), } \\
\text { and Williamson et al. (2013) }\end{array}$ & $\begin{array}{l}\text { Northern blot (Friedländer } \\
\text { et al., 2008, 2009), RT-PCR } \\
\text { (Friedländer et al., 2012) }\end{array}$ & No & Yes & No & $\begin{array}{l}\text { No graphics, } \\
\text { standalone }\end{array}$ \\
\hline miRDeep2 & Bayesian & Bowtie & No & $\begin{array}{l}\text { An et al. (2013), Friedländer et al. } \\
\text { (2012), Hansen et al. (2014), and } \\
\text { Williamson et al. (2013) }\end{array}$ & $\begin{array}{l}\text { Knock-down (Friedländer } \\
\text { et al., 2012), RT-PCR (Metpally } \\
\text { et al., 2013) }\end{array}$ & Yes & Yes & No & $\begin{array}{l}\text { Graphics, } \\
\text { standalone }\end{array}$ \\
\hline miRDeep* & Bayesian & $\begin{array}{l}\text { Bowtie (java } \\
\text { version) }\end{array}$ & No & $\begin{array}{l}\text { An et al. (2013), and Hansen et al. } \\
\text { (2014) }\end{array}$ & $\begin{array}{l}\text { RT-PCR, knock-down (An } \\
\text { et al., 2013) }\end{array}$ & Yes & Yes & TargetScan & $\begin{array}{l}\text { Graphics, } \\
\text { standalone } \\
\text { (java software) }\end{array}$ \\
\hline MIReNA & Rules-based & Megablast & Yes & $\begin{array}{l}\text { An et al. (2013), Friedländer et al. } \\
\text { (2012), and Mathelier and Carbone } \\
\text { (2010) }\end{array}$ & $\begin{array}{l}\text { Knock-down (Friedländer } \\
\text { et al., 2012) }\end{array}$ & No & No & No & No graphics \\
\hline miREvo & Bayesian & Bowtie & No & No & No & Yes & Yes & No & $\begin{array}{l}\text { Graphics, } \\
\text { standalone }\end{array}$ \\
\hline miRExpress & $\begin{array}{l}\text { Sequence } \\
\text { homology }\end{array}$ & $\begin{array}{l}\text { Custom } \\
\text { mapping tools }\end{array}$ & No & No & No & Yes & Yes & No & $\begin{array}{l}\text { No graphics, } \\
\text { standalone }\end{array}$ \\
\hline MiRTRAP & Rules-based & Not included & No & $\begin{array}{l}\text { An et al. (2013), Friedländer et al. } \\
\text { (2012), and Hendrix et al. (2010) }\end{array}$ & $\begin{array}{l}\text { Knock-down (Friedländer } \\
\text { et al., 2012), Northern blot } \\
\text { (Hendrix et al., 2010) }\end{array}$ & No & No & No & No graphics \\
\hline
\end{tabular}




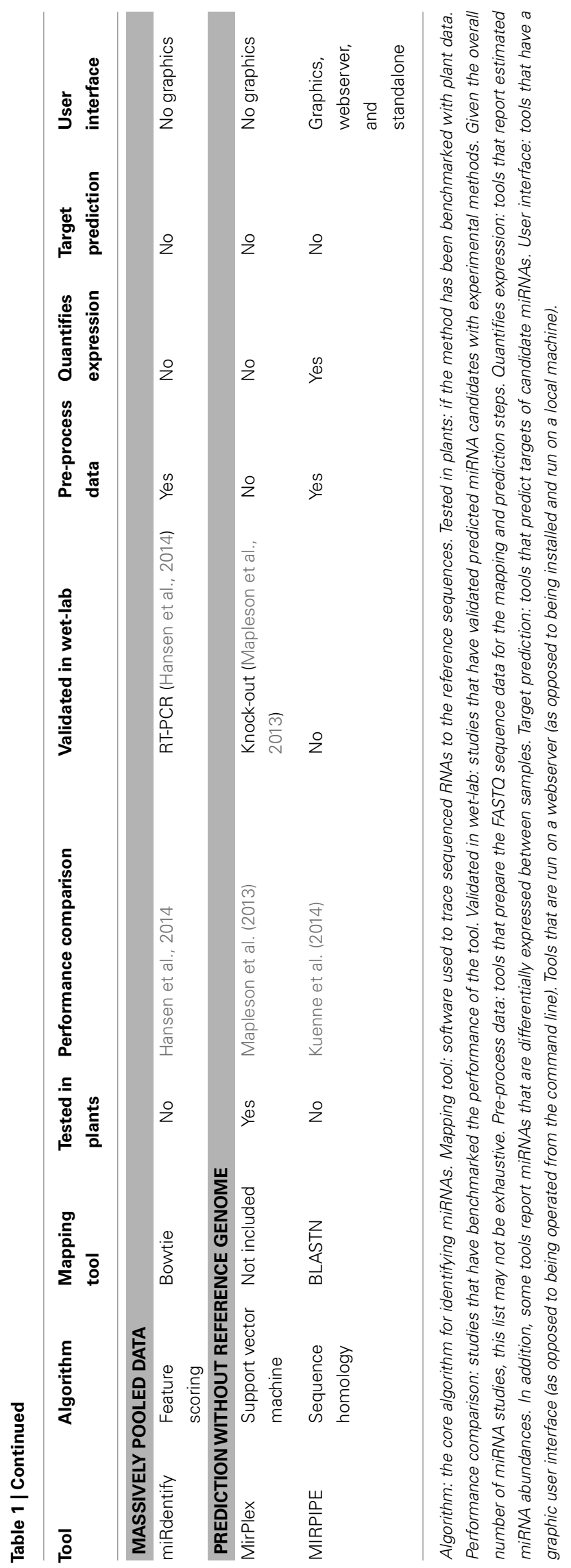

are considered as potential new miRNAs. They are evaluated as miRNAs using a random forest machine learning approach. The classifier is initially trained on a set of known miRNAs from human, rat, or nematode and dozens of features are considered, including energetics, structure, bulges, and the number of reads mapping. The tool has fitted parameters for each species analyzed and on publication provided packages for seven commonly used species. miRanalyzer is available through a webserver, making it easily accessible for biologists with little computational experience ${ }^{2}$.

\section{miRanalyzer (UPDATE)}

miRanalyzer (update) is an improved version with several new features. It uses bowtie (Langmead et al., 2009) for much faster and less memory-intensive mapping, and it includes parameter packages for 31 species, including 6 plants (Hackenberg et al., 2011). In addition, it can perform differential expression analysis of the profiled miRNAs and can predict targets using the TargetSpy tool. In addition to the web server version, it has a stand-alone version that can be downloaded and run on local machines. miRanalyzer predictions have been validated with several wet-lab methods (Smith et al., 2013; Mayoral et al., 2014). Since miRanalyzer often predicts more new miRNAs than do other tools, it is well suited for studies where the predictions will be filtered by additional computational tools or by high-throughput wet-lab validations ${ }^{2}$.

\section{miRCat}

miRCat has been used successfully to predict miRNAs in several plants (Szittya et al., 2008; Pantaleo et al., 2010; Mohorianu et al., 2011) and has recently been adapted to animal sequences, including butterflies (Surridge et al., 2011). miRCat uses a rules-based approach that eliminates candidates with features that are not consistent with miRNA biogenesis (Moxon et al., 2008; Stocks et al., 2012). Numerous features are investigated, including the number of read stacks in the locus, the number of reads mapping anti-sense to the locus, the size of bulges in the candidate miRNA duplex, the number/fraction of paired nucleotides in the duplex and in the hairpin, and the energetic stability of the hairpin. miRCat is part of a suite, the UEA workbench, which includes numerous computational tools, some which can be applied to the analysis of non-miRNA small RNA sequences. miRCat predictions have been validated in several systems (Donaszi-Ivanov et al., 2013; Kohli et al., 2014; Pandey et al., 2014). Since it was developed for plant miRNAs that are more variable in structure, it could be well suited for detecting animal miRNA hairpins that are not typical for this clade $^{3}$.

\section{miRDeep}

miRDeep first filters all candidates whose structure and read signature are inconsistent with Drosha/Dicer processing (Friedländer et al., 2008). In the next step, the fit of the structure and signature to an explicit model of miRNA biogenesis is scored using Bayesian statistics. Specifically, miRDeep scores the number of reads supporting biogenesis, the presence of a miRNA passenger strand, the

\footnotetext{
${ }^{2}$ http://bioinfo2.ugr.es/miRanalyzer/standalone.html

${ }^{3}$ http://srna-workbench.cmp.uea.ac.uk/tools/analysis-tools/mircat/)
} 
presence of a conserved miRNA seed and the absolute and relative energetic stability of the hairpin. While miRDeep can be run on data filtered for known non-miRNA annotations, it can perform robust prediction without this filtering. This means that miRNAs derived from non-canonical host transcripts, such as snoRNAs, can be identified (Ender et al., 2008). Further, it does not require parameters fitted to specific species, meaning that it is not at a disadvantage when mining emerging model systems. The tool has been extensively benchmarked and validated by experimental methods (Friedländer et al., 2008, 2009; Metpally et al., 2013), and has been adapted by several other research groups (Yang and Li, 2011; Yang and Qu, 2012; Wu et al., 2013) ${ }^{4}$.

\section{miRDeep2}

miRDeep2 improves the previous version, primarily by making more robust predictions when faced with very deep sequencing data (Mackowiak, 2011; Friedländer et al., 2012). This includes improved excision of candidate hairpins from the genome, allowing for anti-sense miRNAs and moRs (see miRTRAP below). In addition, the tool has been improved in terms of computational efficiency, implementing better tools like bowtie (Langmead et al., 2009), and it features graphics output. Last, it has been tested in seven species, using the exact same parameters, and introduces knock-down of key proteins necessary for miRNA maturation to validate that novel candidates depend on the miRNA biogenesis pathways for their expression ${ }^{4}$.

\section{miRDeep*}

miRDeep ${ }^{*}$ is an extension of the first miRDeep algorithm, and incorporates many improvements similar to miRDeep2, although it was developed by a separate research group (An et al., 2013). It features pre-processing, bowtie mapping, improved precursor excision, and target prediction for known and novel miRNAs. The tool has an extensive graphical user interface and is implemented entirely in java without requiring any pre-dependent computational tools, making it portable and easy to install. The computational efficiency makes it run on a home computer ${ }^{5}$.

\section{MIReNA}

MIReNA is a flexible tool to predict novel miRNAs from known miRNA sequences, next-generation sequencing data, long transcripts, or hairpin precursors (Mathelier and Carbone, 2010). It uses a rules-based scheme with sharp cut-offs to classify miRNAs based on five criteria: the lack of base pairing in the mature miRNA, the difference in length between the two candidate miRNA strands, the fraction of base-paired nucleotides in the hairpin, and two measures of energetic stability. As a second filtering step, it considers only hairpins where the sequenced RNAs map in consistence with Drosha/Dicer processing. MIReNA can consider several potential miRNA duplexes within one precursor structure, e.g., within multiple stem precursors, giving it the potential to predict non-canonical miRNAs ${ }^{6}$.

\footnotetext{
${ }^{4}$ https://www.mdc-berlin.de/8551903/en/research/research_teams/systems_ biology_of_gene_regulatory_elements/projects/miRDeep

${ }^{5}$ http://www.australianprostatecentre.org/research/software/mirdeep-star

${ }^{6} \mathrm{http}: / /$ www.lgm.upmc.fr/mirena/index.html
}

\section{miREvo}

miREvo build on the miRDeep2 predictor (above) but extends it for evolutionary analyses (Wen et al., 2012). Specifically, it uses whole-genome alignments to identify miRNA homologs in related species. It also includes tools to compare expression of miRNA homologs across species, if sRNA-seq data are available for both species. It uses modified prediction parameters for plant analyses ${ }^{7}$.

\section{miRExpress}

miRExpress is a tool for profiling miRNA expression from sRNAseq data (Wang et al., 2009). However, it includes a function to predict miRNAs based on sequence homology. It maps each read that does not correspond to a known reference miRNA against miRBase sequences, keeping only perfect matches. These reads are then mapped against the reference genome, and the structure evaluated with the mfold structure prediction software (Zuker, $2003)^{8}$

\section{miRTRAP}

miRTRAP uses a rules-based approach with two filtering steps (Hendrix et al., 2010). In the first one, all candidate miRNAs whose structure and read signatures do not conform to Drosha/Dicer processing are eliminated. In the second step, all candidates that are not located in sRNA deserts are removed. This second step builds on the observation that miRNAs typically generates blocks of sRNAs with few or no sequenced RNA mapping to the anti-sense strand or in the general vicinity. In addition to this innovative filtering step, miRTRAP has high accuracy when predicting miRNAs with moRs, which are sRNAs generated from the flanks of the precursor hairpin. This development was necessary, as the tool was initially developed for identifying miRNAs in sea squirt, a species unusually rich in moRs (Shi et al., 2009) ${ }^{9}$.

\section{SPECIAL APPLICATIONS MASSIVELY POOLED DATA}

Many researchers who apply miRNA prediction tools to sequencing data want to mine their own in-house data. These could be sequences from an emerging model organism, or from a human tissue of interest. The tools described above are all optimized for analyzing a limited number of data sets, ranging from maybe 1 to 20 sets. However, some studies compile all the available sRNA-seq data for a given species to give the best possible miRNA annotation. There are numerous advantages to pooling tens or hundreds of datasets (Friedländer et al., 2014). First, if the guide and passenger strands are detected in two distinct data sets, combining the information can allow analysis of the duplex features. Second, lowly expressed miRNAs might not be well profiled in single datasets, where it is difficult to evaluate the read signature. Third, since sRNA-seq library preparation involves a PCR amplification step, there is no guarantee that 10 sequencing reads in 1 dataset do not correspond to a single over-amplified sRNA. In contrast, if the same sequence is detected in data from 10 distinct tissues, this provides independent evidence that the biogenesis is common.

\footnotetext{
${ }^{7}$ http://omictools.com/mirevo-s962.html

${ }^{8}$ http://mirexpress.mbc.nctu.edu.tw

${ }^{9} \mathrm{http} / / /$ flybuzz.berkeley.edu/miRTRAP.html
} 
Massively pooled sRNA-seq data have previously been used to predict miRNAs in general (Friedländer et al., 2014), or of the specific mirtron class (Ladewig et al., 2012). These are hairpins, which are released by intronic splicing rather than Drosha cleavage. Some mirtrons are short and their hairpin ends are defined by the splice signals, while others are longer, and one end is trimmed to define the hairpin end (Berezikov et al., 2007; Okamura et al., 2007; Ruby et al., 2007). In addition, the miRBase database employs massively pooled data to refine the miRNA annotations and define a high-confidence set of sequences (Kozomara and Griffiths-Jones, 2014). The software used in these studies has, however, not been published, so the methods are not described in detail here.

\section{miRdentify}

miRdentify has recently been released to the public to analyze massive pooled data (Hansen et al., 2014). It requires that both guide and passenger miRNA strands are detected and evaluates 10 features of the structure and signature, including precision of $5^{\prime}$ end processing, two nucleotide $3^{\prime}$ overhangs, and several aspect of stability. For each feature, the cut-off is set so that $1 \%$ of known miRNAs is excluded. Together, the requirement for detection of both strands and the 10 features constitute stringent criteria that produce miRNA candidates with features similar to known hairpins (Hansen et al., 2014). The method thus, to some extent, trades off sensitivity to report high-quality candidates ${ }^{10}$.

\section{PREDICTION WITHOUT A REFERENCE GENOME}

The majority of miRNA prediction tools require a reference genome as input to enable the excision of miRNA hairpin sequences, whose RNA structures and signatures are considered as key features for miRNA prediction. However, even though the price of next-generation sequencing technologies decreases, only a handful of model species have fully assembled high-quality reference genomes. Thus, many researchers rely on emerging model species without reference genomes, and novel methods are needed to discover new miRNAs in order to further study their function. One way to address this problem is to use a closely related species genome as proxy reference sequence to identify conserved miRNA. Such a study has been undertaken to discover mosquito miRNAs by mapping the sRNA-seq against the genomes of three related insect species (Etebari and Asgari, 2014). For this purpose, the miRanalyzer tool was used, and it was found that the prediction accuracy is affected by the evolutionary distance between the species of interest and the proxy species. Overall, the most abundant and conserved miRNAs were identified in this study, but the approach might be less successful for species that do not have closely related species with genome sequences.

\begin{abstract}
MirPlex
MirPlex is a tool that requires only sRNA datasets as input with no genome sequences needed (Mapleson et al., 2013). It uses a multi-stage process to identify genuine miRNA duplexes. First, all overlapping sequences are assembled into contigs, and contigs that are too long to be miRNAs are discarded ( $>30$ nucleotides).
\end{abstract}

${ }^{10} \mathrm{http} / / /$ www.ncrnalab.dk/\#mirdentify/mirdentify.php
Second, the remaining sequences are copied into two duplicate datasets followed with separate filter pathways to obtain candidate miRNA guide and miRNA passenger sequences. Last, the candidate miRNA guide and miRNA passenger sequences are then paired into duplexes for the classification. The core algorithm of MirPlex uses a support vector machine to classify genuine miRNA duplexes based on 20 features that divided into three categories: the size of sequences in the duplex, the stability of the duplex, and the nucleotide composition of the duplex. However, MirPlex depends on the presence of both strands in a miRNA duplex for prediction, and so cannot discover miRNAs unless the less abundant passenger strand is also detected by the sequencing ${ }^{11}$.

\section{MIRPIPE}

MIRPIPE identifies miRNAs through sequence homology (Kuenne et al., 2014). It collapses duplicate reads and removes those that have only been sequenced few times. It then further collapses sequences that only differ in the $3^{\prime}$ end and last maps the remaining sequences against known miRBase mature sequences, using the flexible BLAST mapping (Altschul et al., 1990). Since the method relies completely on the presence of known homologs, the prediction accuracy will improve as more miRNAs are deposited to miRBase. However, it cannot identify species-specific miRNAs ${ }^{12}$.

\section{MIRNA VALIDATION NORTHERN BLOT ANALYSIS}

To resolve if a predicted miRNA is genuine, it is often necessary to validate it with methods other than next-generation sequencing. In this respect, Northern blot analysis can be considered as the gold standard (Lee et al., 1993; Ambros et al., 2003). First, the RNA from the cells or tissues of interest is extracted and run on a high-resolution gel. Then, the gel is treated with probes that are complementary in sequence to the predicted miRNA strand. If the strand is expressed in the cells of interest, a band corresponding to 22 nucleotides will show, and in some cases the precursor, which is around 60 nucleotides, will also show. Although this double-band constitutes compelling evidence of miRNA biogenesis, Northern blot analysis has low sensitivity, so many miRNAs that can be reliably profiled by sequencing is below Northern blot detection limit (Table 2).

\section{PCR-BASED METHODS}

In contrast, real-time polymerase chain reaction (RT-PCR) methods can profile and thus validate miRNAs of very low abundance. These methods use sequence-specific primers to bind to the miRNAs and amplify them through reverse transcription and polymerase reaction (Lu et al., 2005). The abundances of amplified sequences are measured by fluorescence, and can be used to estimate the expression of the profiled miRNA. Some systems use stem-loop primers that fold around the $3^{\prime}$ end of the miRNA and can only amplify sequences with that particular end, increasing the specificity of the measurements (Chen et al., 2005). Although RT-PCR methods are considered reliable, the custom primers and probes for newly predicted miRNAs can be costly and the methods are rarely used to validate large sets of sequences.

\footnotetext{
${ }^{11} \mathrm{http}: / /$ www.uea.ac.uk/computing/mirplex

${ }^{12} \mathrm{https} / / /$ bioinformatics.mpi-bn.mpg.de
} 
Table 2 | Methods for miRNA validation

\begin{tabular}{|c|c|c|c|}
\hline Method & Throughput & Pros & Cons \\
\hline Northern blot analysis & Low & $\begin{array}{l}\text { Length of transcripts observed, possibility of } \\
\text { "double-band" }\end{array}$ & Work-intensive, lack of sensitivity \\
\hline PCR-based methods & Low & Specific to transcript $3^{\prime}$ end, sensitive & Costly for large-scale validation \\
\hline Ectopic RNA hairpin expression & Low & miRNA biogenesis is directly tested & $\begin{array}{l}\text { Work-intensive, impractical for large-scale } \\
\text { validation }\end{array}$ \\
\hline $\begin{array}{l}\text { Association with Argonaute } \\
\text { proteins }\end{array}$ & Low/high & Directly shows interaction with effector proteins & Method is not always specific for miRNAs \\
\hline $\begin{array}{l}\text { Inhibition of miRNA biogenesis } \\
\text { pathways }\end{array}$ & Low/high & Directly shows dependence on biogenesis proteins & $\begin{array}{l}\text { Knock-downs are transient and sometimes } \\
\text { weak, generating knock-outs is time-consuming }\end{array}$ \\
\hline $\begin{array}{l}\text { Experimentally identified target } \\
\text { sites }\end{array}$ & Low/high & $\begin{array}{l}\text { Directly demonstrates target interaction or } \\
\text { repression }\end{array}$ & Reporter assays are work-intensive \\
\hline $\begin{array}{l}\text { Conservation and population } \\
\text { selection pressure }\end{array}$ & $\begin{array}{l}\text { Sequence } \\
\text { analysis }\end{array}$ & No wet-lab experiments required & Non-conserved miRNAs can be functional \\
\hline
\end{tabular}

\section{ECTOPIC RNA HAIRPIN EXPRESSION}

In some cases, an miRNA is very lowly expressed, but researchers want to know if the miRNA biogenesis machinery would process it, were it highly expressed. It is possible to synthesize the DNA sequence of the candidate hairpin and clone it into a bacterial or viral vector (Chiang et al., 2010). The vector is then transfected into a cell culture, and the hairpin sequence is expressed. If the hairpin is recognized and cleaved by the miRNA biogenesis machinery, the predicted miRNA strand will accumulate in cells, and can then be detected by less sensitive methods, such as Northern blot analysis. A disadvantage of this method is that it is time-consuming, in that just a few miRNAs can be tested in parallel in one experiment.

\section{ASSOCIATION WITH ARGONAUTE PROTEINS}

Since miRNAs associate with Argonaute proteins, showing that a predicted miRNA interacts with these proteins constitutes strong evidence of its function. There are now anti-bodies for Argonaute proteins in mammals (Ender et al., 2008), meaning that these proteins can be isolated in immuno-precipitation and their associated sRNAs studied. This profiling was previously done by Northern blot analysis or RT-PCR, but is now often done by nextgeneration sequencing, allowing transcriptome-wide validation. In some cases, the interaction between protein and RNA is stabilized by crosslinking (Licatalosi et al., 2008; Hafner et al., 2010), and some studies also investigate interaction with other proteins known to interact with miRNAs, such as DGCR8 (Macias et al., 2012). However, immuno-precipitation studies also have caveats as they are often performed in cell lines, which may not have the same complements of miRNAs as the tissues from which the sequences are sometimes predicted. Further, sRNAs other than miRNAs are sometimes immune-precipitated with Argonaute proteins (Ender et al., 2008), and it is not understood if these reflect genuine biological realities, or rare artifacts introduced during the experiment. Thus, the presence of an miRNA candidate in such an experiment does not constitute final evidence that it is genuine.

\section{INHIBITION OF mIRNA BIOGENESIS PATHWAYS}

It is a hallmark of canonical miRNAs that they depend on the presence of Drosha, Dicer, and DGCR8 for their expression. Thus, if an miRNA candidate is depleted in cells that are void of one or more of these proteins, it constitutes strong evidence that the candidate is genuine. The expression of the proteins can be knocked down through RNA interference, where artificial sRNAs complementary in sequence to the Drosha, Dicer, or DGCR8 mRNAs are introduced into cells (Friedländer et al., 2012, 2014). The sRNAs can bind to the mRNAs and reduce protein output transiently. The genes can also be conditionally knocked out using genetic methods (Babiarz et al., 2008). In this case, Drosha, Dicer, or DGCR8 genes are deleted, leading to a collapse of the miRNA populations. Both with RNA interference and genetic methods, it is possible to use next-generation sequencing to profile miRNA expression transcriptome-wide before and after the loss of the biogenesis pathways. A limitation of the knock-down approach is that effects on the sRNA expression level are often subtle and transient (Friedländer et al., 2012). The genetic knock-outs give much clearer results, but require generation of mutant animals or cells, which is not trivial, even with the advances made with the CRISPR/Cas9 system (Cong et al., 2013; Mali et al., 2013).

\section{EXPERIMENTALLY IDENTIFIED TARGET SITES}

Arguably, demonstrating the function of a miRNA constitutes stronger evidence than demonstrating its biogenesis or association with proteins. For this purpose, reporter constructs can be designed that are fusions of a target $3^{\prime}$ UTR and a reporter gene that express a marker such as luciferase (Zeng and Cullen, 2003). If the fluorescence is specifically reduced in the presence of the guide miRNA, this indicates an miRNA-target interaction. These reporter assays can be designed to simulate natural cell conditions, with endogenous miRNA and target levels and a natural number of target sites. While this method is time-consuming and only tests a single miRNA in one experiment, new genomics data can profile miRNA-target interaction transcriptome-wide (Helwak et al., 
2013; Grosswendt et al., 2014). These methods use exogenous or endogenous ligases to crosslink miRNAs and their targets, and subsequently sequence these chimeric sequences, yielding information on miRNA-target pairs. These data have been found to contain novel miRNA candidates linked to mRNA sites that have typical target features (Friedländer et al., 2014).

\section{CONSERVATION AND POPULATION SELECTION PRESSURE}

Some miRNAs, like let-7, are deeply conserved and retain almost the exact same sequence in all animals with bilateral body types, ranging from nematode to fruit fly to human (Pasquinelli et al., 2000). Thus miRNA validation is transitive: if a validated miRNA is conserved in a new species, it is likely to be genuine. There are numerous criteria for defining if an miRNA is conserved, but some parts are more likely to be under negative selection. Often homologous genome sequences from numerous species are aligned and the conservation studied to see which parts are most conserved. The nucleotides $2-8$ in the $5^{\prime}$ end of the miRNA (the "seed") are important for target specificity and are often conserved in evolution (Lai, 2002). In fact, miRNAs are grouping into functional gene families based on their seed sequence. The remaining part of the miRNA guide strand also confers binding specificity (Bartel, 2009) and the passenger strand is important for forming duplex with the guide. Last, the sequences flanking the two miRNA strands often exhibit some conservation, as these regions are important for the hairpin structure, and for recruiting proteins during biogenesis (Han et al., 2006). There are examples of miRNAs that are speciesspecific, yet have well-defined and important functions (Hu et al., 2012). In these cases, cross-species conservation patterns cannot be used, but intra-species population studies can reveal selection pressures (Friedländer et al., 2014). However, since these selection pressures can be very subtle, large numbers of novel miRNA genes are needed to detect trends, so the population approaches are not applicable to most studies. Further, sequences can to some extent be conserved by chance, so it often does not constitute definite evidence of function.

\section{COMPUTATIONAL BENCHMARKING}

Wet-lab experiments include gold standards for demonstrating that a given miRNA candidate is genuine. But computational benchmarking can give some estimates to the performance of methods to predict miRNAs, and can compare strengths and weaknesses of distinct algorithms. An advantage of benchmarking is further that it is easily undertaken by computational research groups, while performing Northern blot analyses, for instance, may require substantial investment of time and funds.

Some of the most widely used measures of prediction performance are sensitivity, specificity, and accuracy (Table 3). Sensitivity is the fraction of known distinct miRNAs in the data that are recovered by the method. Specificity is the fraction of (assumed) non-miRNA sequences that are correctly discarded by the algorithm. The false positive rate is the fraction of non-miRNA sequences that are incorrectly reported as miRNAs, or 1 - sensitivity. Accuracy is the fraction of distinct sequences that are correctly classified by the method, summing over all miRNAs and nonmiRNAs. Another common measure of prediction performance is the area under curve (AUC) of receiver operating characteristic
Table 3 | Sensitivity, specificity, and accuracy.

miRNA state

\begin{tabular}{llll} 
& & Genuine miRNA & Not genuine miRNA \\
\cline { 3 - 3 } miRNA & Positive & True positives (TP) & False positives (FP) \\
prediction & Negative & False negatives (FN) & True negatives (TN) \\
Formulas & $\begin{array}{l}\text { Sensitivity or true positive rate } \\
\text { Specificity or true negative rate }\end{array}$ & $\mathrm{TP} /(\mathrm{TP}+\mathrm{FN})$ \\
& $\begin{array}{l}\mathrm{T} /(\mathrm{FP}+\mathrm{TN}) \\
\text { Accuracy }\end{array}$ & $(\mathrm{TP}+\mathrm{TN}) /(\mathrm{TP}+\mathrm{FP}+\mathrm{FN}+\mathrm{TN})$
\end{tabular}

(ROC) Curve. The sensitivity is plotted as a function of the false positive rate, showing the trade-off between sensitivity and specificity. The area under the curve indicates performance, with the full area $(100 \%)$ corresponding to perfect prediction, while half area (50\%) corresponding to prediction that is no better than random.

However, the problem of predicting miRNAs from sRNA-seq data is often a skewed one. That is, if tens of thousands of candidate hairpins are being investigated, the number of genuine miRNA precursors is typically in the hundreds. In other words, the number of negatives often vastly outnumbers the positives. Therefore, a modest reduction in sensitivity can often be tolerated, while a modest reduction in specificity can result in an unmanageable number of false positives. For instance, a reduction in sensitivity from 99 to $90 \%$ will mean a $9 \%$ loss of genuine miRNAs, while a corresponding reduction in specificity will cause a 10 -fold increase in false positives, potentially rendering the resulting predictions useless. To address this, true positives and false positives are often reported as absolute numbers, to give a concrete idea of the number of sequences a user of the methods will encounter. Some methods, like miRDeep and miRDeep2, include computational controls to give the user an idea of the number of false positives generated by each run.

Most studies presenting tools to predict miRNA genes include benchmarking of their own method, often comparing it to competitor methods. A summary of these comparisons would be too comprehensive for this review; however, we have listed all the benchmarking in Table 1. However, two independent studies have been undertaken to compare the prediction performance of miRNA discovery tools. One study found miRExpress to be the most sensitive method and the mirTools suite (which uses miRDeep for prediction) to be the most accurate method (Li et al., 2012). However, we caution against relying too much on the findings of this study, as the inferred performance of the distinct tools differs widely from other performance comparisons (as referenced in Table 1). Another independent study has been undertaken to compare the prediction performance of miRDeep, miRDeep2, and miRanalyzer (updated version), which are some of the most widely used methods in the field (Williamson et al., 2013). One tool, DSAP, which quantifies miRNAs in sRNA-seq was also included in the study, but is not described here as it does not predict new miRNAs. The tools were tested against six biological datasets from cell lines and one simulated negative control data set. miRDeep2 was overall found to have the highest sensitivity, while miRanalyzer reported the most novel miRNA candidates. However, it 
also reported miRNAs from the simulated data, suggesting that some of the ones reported from the biological data are false positives. miRDeep had the best overall trade-off between sensitivity and specificity, as measured by AUC, followed by miRDeep2. It should be mentioned that this benchmarking just represents performance in a few use cases, and more independent studies should be undertaken to evaluate the strengths and weaknesses of the existing methods.

\section{VISUAL INSPECTION OF STRUCTURE AND READ SIGNATURE}

Many tools for miRNA prediction generate graphics of the novel candidates, showing the RNA structure and the positions of the sequenced RNAs relative to the hairpin. With experience, it is possible to make estimates which of the novel candidate miRNAs can be validated in wet-lab experiments, and which will turn out to be false positive predictions. The human eye is a sensitive tool that can discriminate subtle features that are difficult to score computationally without loss of sensitivity. For instance, the miRNA hairpin structure will rarely contain large bulges, but will also rarely form a tight stem. Also, the processing of miRNA $5^{\prime}$ ends tends to be more precise than processing of the $3^{\prime}$ end (Ruby et al., 2006). Spending some time looking at gold standard known miRNAs can teach a researcher to identify these and more features. Of course, visual inspection of structure and read signature is no substitute for validation, but it can give the trained miRNA researcher an estimate of the quality of his predictions.

\section{FUTURE DIRECTIONS OF THE FIELD RESOLVING AMBIGUOUS SEQUENCES}

Any miRNA prediction depends on read mappings that trace the sequenced RNAs to the genome loci from which they were transcribed. sRNA-seq presents difficulties that are rarely encountered in mRNA sequencing. We know from biology that each deep sequenced RNA has been transcribed from exactly one genome locus. However, when sequenced sRNAs are mapped to the reference genome, many map to more than one locus. This is in some cases because the RNA is transcribed from a gene with many copies in the genome, like a transposable element. In some cases, it will be "spurious" mappings, meaning that a short sequence can have chance matches to biologically unrelated positions in the genome, especially when the reference genome is large. A solution to the problem could be to assume that most deep sequencing reads have originated from a relatively small number of genome loci, and attempt to map the reads such that most of them locate to the fewest possible number of loci. In some concrete cases, this appears reasonable. For instance, imagine a read that maps equally well to two genome loci. One locus is a "read desert" with no other reads mapping nearby. The other locus is an rRNA gene that has thousands of reads mapping. In this case, it would seem reasonable to assume that the read should be mapped to the highly expressed rRNA locus. Some work has already been made toward overcoming these challenges. The tool SeqCluster first fuses reads that overlap in sequence in a tiled way, and subsequently maps the fused sequences to the genome (Pantano et al., 2011). These methods can resolve many, although not all, ambiguous mappings.

\section{CROSS-MAPPING EVENTS}

Even though next-generation sequencing quality has improved the last years, some nucleotides are inevitably called incorrectly. Similarly, sRNAs can undergo biological editing events or have untemplated nucleotides added to their $3^{\prime}$ ends. In these cases, an sRNA will no longer map perfectly to the genome position; it was originally transcribed from, but it may map perfectly to a distinct genome position (de Hoon et al., 2010). These wrongly mapped sRNAs will often be considered by miRNA prediction algorithms and may cause false positives. In one study, an explicit statistical model to correct these errors was developed, and numerous wrong mappings were corrected (de Hoon et al., 2010). However, this model has to our knowledge never been implemented as a user-friendly mapping tool. Ideally, such a model could be combined with a method to unambiguously trace sequenced RNAs to a single genome position (above). This would provide the sRNA community with a custom tool to handle some of the difficulties inherent in studying short sequences, and would provide an excellent platform for miRNA prediction.

\section{REPEAT-DERIVED miRNAs}

The most commonly used tools for miRNA prediction discards mature sequences that map to many genome loci. This is a practical step to reduce the number of genome loci investigated and thus the number of false positives. However, it is well established that miRNA hairpins can arise from repetitive sequences such as transposable elements (Smalheiser and Torvik, 2005; Berezikov, 2011), and these cannot be detected by current prediction methods, unless the hairpins have diverged in sequence from the consensus repeats. Since repeat-derived sRNAs have been shown to have important functions in, for instance, the mammalian germ line (Aravin et al., 2006; Girard et al., 2006; Grivna et al., 2006; Lau et al., 2006; Watanabe et al., 2006, 2008; Tam et al., 2008), it would be interesting to investigate the prevalence and function of repeatderived miRNAs. However, such a study could be complicated by multi-mapping problems (above) and would be much facilitated by the development of custom mapping and sequence analysis tools. Overall, the field of mapping sRNAs is understudied, and advances in this field could benefit the community.

\section{REDUCING sRNA-seq BIASES}

It is well established that library preparation introduces strong biases in sRNA-seq. One study has shown that artificial miRNAs introduced to a buffer in carefully controlled equal abundance give rise to numbers of reads that differ by orders of magnitude (Linsen et al., 2009). This means that some miRNAs give rise to disproportionate large numbers of reads, while others are difficult to detect and thus also more difficult to discover using sequencing. A recent study has traced these biases back to the ligase protein that joins the miRNA with sequencing adapters (Sorefan et al., 2012). miRNAs and adapters together form structures, some of which are easily ligated and some of which are difficult to ligate. In fact, since most sRNA-seq studies use the same ligase and the same adapters (from the Illumina small RNA TruSeq protocol), the miRBase database has been biased toward miRNAs that are easily ligated with this protocol. The researchers of this study has developed an alternative "high definition" protocol using pools of 
adapters that even out the biases, giving a more even representation of miRNAs and facilitating identification of novel sequences (Sorefan et al., 2012). As this protocol becomes more widely used in miRNAs discovery efforts, the skew in the miRBase database will, for sure, be corrected.

\section{UNDERSTANDING THE FEATURES THAT DETERMINE HAIRPIN BIOGENESIS}

The human transcriptome contains more than 100,000 hairpin structures that resemble miRNA precursors (unpublished results). More than half of these are located in protein coding transcripts. Thus, while many mRNAs and miRNA primary transcripts resemble each other in being capped, poly-adenylated, and containing hairpin structures, the mRNAs are transported to the cytosol and translated, while the pri-miRNAs are cleaved into regulatory sRNAs. This mystery underlines our incomplete understanding of miRNA biogenesis: which features determine if a given hairpin is cleaved into miRNAs or left untouched? Does the presence of protein factors protect the hairpin or make it available for Drosha processing? Or does protein competition determine the hairpin fate? And which structural and sequence features of the hairpin determine which proteins are bound? Studies are unraveling these interactions (Auyeung et al., 2013) but it is clear that our understanding is still incomplete. If we would understand what hairpin features license biogenesis, we would be able to computationally predict from genome sequence, which hairpins are cleaved to miRNAs and which are left untouched.

\section{ACKNOWLEDGMENTS}

Wenjing Kang and Marc Riemer Friedländer acknowledge funding from the Strategic Research Area program of the Swedish Research Council through Stockholm University.

\section{REFERENCES}

Abelson, J. F., Kwan, K. Y., O’Roak, B. J., Baek, D. Y., Stillman, A. A., Morgan, T. M., et al. (2005). Sequence variants in SLITRK1 are associated with Tourette's syndrome. Science 310, 317-320. doi:10.1126/science.1116502

Altschul, S. F., Gish, W., Miller, W., Myers, E. W., and Lipman, D. J. (1990). Basic local alignment search tool. J. Mol. Biol. 215, 403-410. doi:10.1016/S0022-2836(05) 80360-2

Ambros, V., Bartel, B., Bartel, D. P., Burge, C. B., Carrington, J. C., Chen, X., et al. (2003). A uniform system for microRNA annotation. RNA 9, 277-279. doi:10.1261/rna.2183803

An, J., Lai, J., Lehman, M. L., and Nelson, C. C. (2013). miRDeep*: an integrated application tool for miRNA identification from RNA sequencing data. Nucleic Acids Res. 41, 727-737. doi:10.1093/nar/gks1187

Aravin, A., Gaidatzis, D., Pfeffer, S., Lagos-Quintana, M., Landgraf, P., Iovino, N., et al. (2006). A novel class of small RNAs bind to MILI protein in mouse testes. Nature 442, 203-207. doi:10.1038/nature04916

Auyeung, V. C., Ulitsky, I., Mcgeary, S. E., and Bartel, D. P. (2013). Beyond secondary structure: primary-sequence determinants license pri-miRNA hairpins for processing. Cell 152, 844-858. doi:10.1016/j.cell.2013.01.031

Babiarz, J. E., Ruby, J. G., Wang, Y., Bartel, D. P., and Blelloch, R. (2008). Mouse ES cells express endogenous shRNAs, siRNAs, and other microprocessorindependent, dicer-dependent small RNAs. Genes Dev. 22, 2773-2785. doi:10. 1101/gad.1705308

Bartel, D. P. (2009). microRNAs: target recognition and regulatory functions. Cell 136, 215-233. doi:10.1016/j.cell.2009.01.002

Bentwich, I. (2005). Prediction and validation of microRNAs and their targets. FEBS Lett. 579, 5904-5910. doi:10.1016/j.febslet.2005.09.040

Berezikov, E. (2011). Evolution of microRNA diversity and regulation in animals. Nat. Rev. Genet. 12, 846-860. doi:10.1038/nrg3079
Berezikov, E., Chung, W. J., Willis, J., Cuppen, E., and Lai, E. C. (2007). Mammalian mirtron genes. Mol. Cell 28, 328-336. doi:10.1016/j.molcel.2007.09.028

Berezikov, E., Guryev, V., Van De Belt, J., Wienholds, E., Plasterk, R. H., and Cuppen, E. (2005). Phylogenetic shadowing and computational identification of human microRNA genes. Cell 120, 21-24. doi:10.1016/j.cell.2004.12.031

Berninger, P., Gaidatzis, D., Van Nimwegen, E., and Zavolan, M. (2008). Computational analysis of small RNA cloning data. Methods 44, 13-21. doi:10.1016/j. ymeth.2007.10.002

Bernstein, E., Caudy, A. A., Hammond, S. M., and Hannon, G. J. (2001). Role for a bidentate ribonuclease in the initiation step of RNA interference. Nature 409, 363-366. doi:10.1038/35053110

Bernstein, E., Kim, S. Y., Carmell, M. A., Murchison, E. P., Alcorn, H., Li, M. Z., et al. (2003). Dicer is essential for mouse development. Nat. Genet. 35, 215-217. doi:10.1038/ng1253

Bushati, N., and Cohen, S. M. (2007). microRNA functions. Annu. Rev. Cell Dev. Biol. 23, 175-205. doi:10.1146/annurev.cellbio.23.090506.123406

Calabrese, J. M., Seila, A. C., Yeo, G. W., and Sharp, P. A. (2007). RNA sequence analysis defines dicer's role in mouse embryonic stem cells. Proc. Natl. Acad. Sci. U.S.A. 104, 18097-18102. doi:10.1073/pnas.0709193104

Chen, C., Ridzon, D. A., Broomer, A. J., Zhou, Z., Lee, D. H., Nguyen, J. T., et al. (2005). Real-time quantification of microRNAs by stem-loop RT-PCR. Nucleic Acids Res. 33, e179. doi:10.1093/nar/gnil78

Chen, C. J., and Heard, E. (2013). Small RNAs derived from structural non-coding RNAs. Methods 63, 76-84. doi:10.1016/j.ymeth.2013.05.001

Chiang, H. R., Schoenfeld, L. W., Ruby, J. G., Auyeung, V. C., Spies, N., Baek, D., et al. (2010). Mammalian microRNAs: experimental evaluation of novel and previously annotated genes. Genes Dev. 24, 992-1009. doi:10.1101/gad. 1884710

Christensen, B. C., Moyer, B. J., Avissar, M., Ouellet, L. G., Plaza, S. L., Mcclean, M. D., et al. (2009). A let-7 microRNA-binding site polymorphism in the KRAS $3^{\prime}$ UTR is associated with reduced survival in oral cancers. Carcinogenesis 30, 1003-1007. doi:10.1093/carcin/bgp099

Cimmino, A., Calin, G. A., Fabbri, M., Iorio, M. V., Ferracin, M., Shimizu, M., et al. (2005). miR-15 and miR-16 induce apoptosis by targeting BCL2. Proc. Natl. Acad. Sci. U.S.A. 102, 13944-13949. doi:10.1073/pnas.0506654102

Cong, L., Ran, F. A., Cox, D., Lin, S., Barretto, R., Habib, N., et al. (2013). Multiplex genome engineering using CRISPR/Cas systems. Science 339, 819-823. doi:10.1126/science. 1231143

Davalos, V., Moutinho, C., Villanueva, A., Boque, R., Silva, P., Carneiro, F., et al. (2012). Dynamic epigenetic regulation of the microRNA-200 family mediates epithelial and mesenchymal transitions in human tumorigenesis. Oncogene 31, 2062-2074. doi:10.1038/onc.2011.383

de Hoon, M. J., Taft, R. J., Hashimoto, T., Kanamori-Katayama, M., Kawaji, H., Kawano, M., et al. (2010). Cross-mapping and the identification of editing sites in mature microRNAs in high-throughput sequencing libraries. Genome Res. 20, 257-264. doi:10.1101/gr.095273.109

Denli, A. M., Tops, B. B., Plasterk, R. H., Ketting, R. F., and Hannon, G. J. (2004). Processing of primary microRNAs by the microprocessor complex. Nature 432, 231-235. doi:10.1038/nature03049

Dezulian, T., Remmert, M., Palatnik, J. F., Weigel, D., and Huson, D. H. (2006). Identification of plant microRNA homologs. Bioinformatics 22, 359-360. doi 10.1093/bioinformatics/bti802

Donaszi-Ivanov, A., Mohorianu, I., Dalmay, T., and Powell, P. P. (2013). Small RNA analysis in Sindbis virus infected human HEK293 cells. PLoS One 8:e84070. doi:10.1371/journal.pone.0084070

Ender, C., Krek, A., Friedländer, M. R., Beitzinger, M., Weinmann, L., Chen, W., et al. (2008). A human snoRNA with microRNA-like functions. Mol. Cell 32, 519-528. doi:10.1016/j.molcel.2008.10.017

Esteller, M. (2011). Non-coding RNAs in human disease. Nat. Rev. Genet. 12, 861-874. doi:10.1038/nrg3074

Etebari, K., and Asgari, S. (2014). Accuracy of microRNA discovery pipelines in non-model organisms using closely related species genomes. PLoS One 9:e84747. doi:10.1371/journal.pone.0084747

Farazi, T. A., Juranek, S. A., and Tuschl, T. (2008). The growing catalog of small RNAs and their association with distinct Argonaute/Piwi family members. Development 135, 1201-1214. doi:10.1242/dev.005629

Filipowicz, W., Bhattacharyya, S. N., and Sonenberg, N. (2008). Mechanisms of post-transcriptional regulation by microRNAs: are the answers in sight? Nat. Rev. Genet. 9, 102-114. doi:10.1038/nrg2290 
Friedländer, M. R., Adamidi, C., Han, T., Lebedeva, S., Isenbarger, T. A., Hirst, M., et al. (2009). High-resolution profiling and discovery of planarian small RNAs. Proc. Natl. Acad. Sci. U.S.A. 106, 11546-11551. doi:10.1073/pnas.0905222106

Friedländer, M. R., Chen, W., Adamidi, C., Maaskola, J., Einspanier, R., Knespel, S. et al. (2008). Discovering microRNAs from deep sequencing data using miRDeep. Nat. Biotechnol. 26, 407-415. doi:10.1038/nbt1394

Friedländer, M. R., Lizano, E., Houben, A. J., Bezdan, D., Banez-Coronel, M., Kudla, G., et al. (2014). Evidence for the biogenesis of more than 1,000 novel human microRNAs. Genome Biol. 15, R57. doi:10.1186/gb-2014-15-4-r57

Friedländer, M. R., Mackowiak, S. D., Li, N., Chen, W., and Rajewsky, N. (2012). miRDeep2 accurately identifies known and hundreds of novel microRNA genes in seven animal clades. Nucleic Acids Res. 40, 37-52. doi:10.1093/nar/gkr688

Friedman, R. C., Farh, K. K., Burge, C. B., and Bartel, D. P. (2009). Most mammalian mRNAs are conserved targets of microRNAs. Genome Res. 19, 92-105. doi:10.1101/gr.082701.108

Ghildiyal, M., and Zamore, P. D. (2009). Small silencing RNAs: an expanding universe. Nat. Rev. Genet. 10, 94-108. doi:10.1038/nrg2504

Giraldez, A. J., Cinalli, R. M., Glasner, M. E., Enright, A. J., Thomson, J. M., Baskerville, S., et al. (2005). microRNAs regulate brain morphogenesis in zebrafish. Science 308, 833-838. doi:10.1126/science.1109020

Girard, A., Sachidanandam, R., Hannon, G. J., and Carmell, M. A. (2006). A germline-specific class of small RNAs binds mammalian Piwi proteins. Nature 442, 199-202. doi:10.1038/nature04917

Gregory, R. I., Yan, K. P., Amuthan, G., Chendrimada, T., Doratotaj, B., Cooch, N., et al. (2004). The microprocessor complex mediates the genesis of microRNAs. Nature 432, 235-240. doi:10.1038/nature03120

Grivna, S. T., Beyret, E., Wang, Z., and Lin, H. (2006). A novel class of small RNAs in mouse spermatogenic cells. Genes Dev. 20, 1709-1714. doi:10.1101/gad.1434406

Grosswendt, S., Filipchyk, A., Manzano, M., Klironomos, F., Schilling, M., Herzog, M., et al. (2014). Unambiguous identification of miRNA:target site interactions by different types of ligation reactions. Mol. Cell 54, 1042-1054. doi:10.1016/j.molcel.2014.03.049

Guo, L., and Lu, Z. (2010). The fate of miRNA* strand through evolutionary analysis: implication for degradation as merely carrier strand or potential regulatory molecule? PLoS One 5:e11387. doi:10.1371/journal.pone.0011387

Ha, M., and Kim, V. N. (2014). Regulation of microRNA biogenesis. Nat. Rev. Mol. Cell Biol. 15, 509-524. doi:10.1038/nrm3838

Hackenberg, M., Rodriguez-Ezpeleta, N., and Aransay, A. M. (2011). miRanalyzer: an update on the detection and analysis of microRNAs in high-throughput sequencing experiments. Nucleic Acids Res. 39, W132-W138. doi:10.1093/nar/gkr247

Hackenberg, M., Sturm, M., Langenberger, D., Falcon-Perez, J. M., and Aransay, A. M. (2009). miRanalyzer: a microRNA detection and analysis tool for nextgeneration sequencing experiments. Nucleic Acids Res. 37, W68-W76. doi:10. 1093/nar/gkp347

Hafner, M., Landthaler, M., Burger, L., Khorshid, M., Hausser, J., Berninger, P., et al. (2010). Transcriptome-wide identification of RNA-binding protein and microRNA target sites by PAR-CLIP. Cell 141, 129-141. doi:10.1016/j.cell.2010. 03.009

Han, J., Lee, Y., Yeom, K. H., Kim, Y. K., Jin, H., and Kim, V. N. (2004). The DroshaDGCR8 complex in primary microRNA processing. Genes Dev. 18, 3016-3027. doi:10.1101/gad.1262504

Han, J., Lee, Y., Yeom, K. H., Nam, J. W., Heo, I., Rhee, J. K., et al. (2006). Molecular basis for the recognition of primary microRNAs by the Drosha-DGCR8 complex. Cell 125, 887-901. doi:10.1016/j.cell.2006.03.043

Hansen, T. B., Veno, M. T., Kjems, J., and Damgaard, C. K. (2014). miRdentify: high stringency miRNA predictor identifies several novel animal miRNAs. Nucleic Acids Res. 42, e124. doi:10.1093/nar/gku598

He, L., Thomson, J. M., Hemann, M. T., Hernando-Monge, E., Mu, D., Goodson, S., et al. (2005). A microRNA polycistron as a potential human oncogene. Nature 435, 828-833. doi:10.1038/nature03552

Helwak, A., Kudla, G., Dudnakova, T., and Tollervey, D. (2013). Mapping the human miRNA interactome by CLASH reveals frequent noncanonical binding. Cell 153, 654-665. doi:10.1016/j.cell.2013.03.043

Hendrix, D., Levine, M., and Shi, W. (2010). miRTRAP, a computational method for the systematic identification of miRNAs from high throughput sequencing data. Genome Biol. 11, R39. doi:10.1186/gb-2010-11-4-r39

Hill, D. A., Ivanovich, J., Priest, J. R., Gurnett, C. A., Dehner, L. P., Desruisseau, D., et al. (2009). DICER1 mutations in familial pleuropulmonary blastoma. Science 325, 965. doi:10.1126/science.1174334
Hu, H. Y., He, L., Fominykh, K., Yan, Z., Guo, S., Zhang, X., et al. (2012). Evolution of the human-specific microRNA miR-941. Nat. Commun. 3, 1145. doi:10.1038/ncomms2146

Huntzinger, E., and Izaurralde, E. (2011). Gene silencing by microRNAs: contributions of translational repression and mRNA decay. Nat. Rev. Genet. 12, 99-110. doi:10.1038/nrg2936

Hutvagner, G., Mclachlan, J., Pasquinelli, A. E., Balint, E., Tuschl, T., and Zamore, P. D. (2001). A cellular function for the RNA-interference enzyme dicer in the maturation of the let-7 small temporal RNA. Science 293, 834-838. doi:10.1126/science.1062961

Jiang, P., Wu, H., Wang, W., Ma, W., Sun, X., and Lu, Z. (2007). MiPred: classification of real and pseudo microRNA precursors using random forest prediction model with combined features. Nucleic Acids Res. 35, W339-W344. doi:10.1093/nar/gkm368

Johnston, R. J., and Hobert, O. (2003). A microRNA controlling left/right neuronal asymmetry in Caenorhabditis elegans. Nature 426, 845-849. doi:10.1038/ nature 02255

Ketting, R. F., Fischer, S. E., Bernstein, E., Sijen, T., Hannon, G. J., and Plasterk, R. H. (2001). Dicer functions in RNA interference and in synthesis of small RNA involved in developmental timing in C. elegans. Genes Dev. 15, 2654-2659. doi:10.1101/gad.927801

Kloosterman, W. P., and Plasterk, R. H. (2006). The diverse functions of microRNAs in animal development and disease. Dev. Cell 11, 441-450. doi:10.1016/j.devcel. 2006.09.009

Knight, S. W., and Bass, B. L. (2001). A role for the RNase III enzyme DCR-1 in RNA interference and germ line development in Caenorhabditis elegans. Science 293, 2269-2271. doi:10.1126/science.1062039

Kohli, D., Joshi, G., Deokar, A. A., Bhardwaj, A. R., Agarwal, M., Katiyar-Agarwal, S., et al. (2014). Identification and characterization of wilt and salt stressresponsive microRNAs in chickpea through high-throughput sequencing. PLoS One 9:e108851. doi:10.1371/journal.pone.0108851

Kozomara, A., and Griffiths-Jones, S. (2011). miRBase: integrating microRNA annotation and deep-sequencing data. Nucleic Acids Res. 39, D152-D157. doi:10.1093/nar/gkq1027

Kozomara, A., and Griffiths-Jones, S. (2014). miRBase: annotating high confidence microRNAs using deep sequencing data. Nucleic Acids Res. 42, D68-D73. doi:10.1093/nar/gkt1181

Kuenne, C., Preussner, J., Herzog, M., Braun, T., and Looso, M. (2014). MIRPIPE: quantification of microRNAs in niche model organisms. Bioinformatics 30, 3412-3413. doi:10.1093/bioinformatics/btu573

Ladewig, E., Okamura, K., Flynt, A. S., Westholm, J. O., and Lai, E. C. (2012). Discovery of hundreds of mirtrons in mouse and human small RNA data. Genome Res. 22, 1634-1645. doi:10.1101/gr.133553.111

Lagos-Quintana, M., Rauhut, R., Lendeckel, W., and Tuschl, T. (2001). Identification of novel genes coding for small expressed RNAs. Science 294, 853-858. doi:10.1126/science.1064921

Lai, E. C. (2002). microRNAs are complementary to $3^{\prime}$ UTR sequence motifs that mediate negative post-transcriptional regulation. Nat. Genet. 30, 363-364. doi: $10.1038 / \mathrm{ng} 865$

Lai, E. C., Tomancak, P., Williams, R. W., and Rubin, G. M. (2003). Computational identification of Drosophila microRNA genes. Genome Biol. 4, R42. doi:10.1186/gb-2003-4-7-r42

Landthaler, M., Yalcin, A., and Tuschl, T. (2004). The human DiGeorge syndrome critical region gene 8 and its D. melanogaster homolog are required for miRNA biogenesis. Curr. Biol. 14, 2162-2167. doi:10.1016/j.cub.2004.11.001

Langenberger, D., Pundhir, S., Ekstrom, C. T., Stadler, P. F., Hoffmann, S., and Gorodkin, J. (2012). deepBlockAlign: a tool for aligning RNA-seq profiles of read block patterns. Bioinformatics 28, 17-24. doi:10.1093/bioinformatics/btr598

Langmead, B., Trapnell, C., Pop, M., and Salzberg, S. L. (2009). Ultrafast and memory-efficient alignment of short DNA sequences to the human genome. Genome Biol. 10, R25. doi:10.1186/gb-2009-10-3-r25

Lau, N. C., Lim, L. P., Weinstein, E. G., and Bartel, D. P. (2001). An abundant class of tiny RNAs with probable regulatory roles in Caenorhabditis elegans. Science 294, 858-862. doi:10.1126/science.1065062

Lau, N. C., Seto, A. G., Kim, J., Kuramochi-Miyagawa, S., Nakano, T., Bartel, D. P., et al. (2006). Characterization of the piRNA complex from rat testes. Science 313, 363-367. doi:10.1126/science.1130164

Lee, R. C., and Ambros, V. (2001). An extensive class of small RNAs in Caenorhabditis elegans. Science 294, 862-864. doi:10.1126/science.1065329 
Lee, R. C., Feinbaum, R. L., and Ambros, V. (1993). The C. elegans heterochronic gene lin-4 encodes small RNAs with antisense complementarity to lin-14. Cell 75, 843-854. doi:10.1016/0092-8674(93)90529-Y

Li, Y., Zhang, Z., Liu, F., Vongsangnak, W., Jing, Q., and Shen, B. (2012). Performance comparison and evaluation of software tools for microRNA deep-sequencing data analysis. Nucleic Acids Res. 40, 4298-4305. doi:10.1093/nar/gks043

Licatalosi, D. D., Mele, A., Fak, J. J., Ule, J., Kayikci, M., Chi, S. W., et al. (2008). HITS-CLIP yields genome-wide insights into brain alternative RNA processing. Nature 456, 464-469. doi:10.1038/nature07488

Lim, L. P., Lau, N. C., Weinstein, E. G., Abdelhakim, A., Yekta, S., Rhoades, M. W., et al. (2003). The microRNAs of Caenorhabditis elegans. Genes Dev. 17,991-1008. doi:10.1101/gad.1074403

Linsen, S. E., De Wit, E., Janssens, G., Heater, S., Chapman, L., Parkin, R. K., et al. (2009). Limitations and possibilities of small RNA digital gene expression profiling. Nat. Methods 6, 474-476. doi:10.1038/nmeth0709-474

Lu, D. P., Read, R. L., Humphreys, D. T., Battah, F. M., Martin, D. I., and Rasko, J. E. (2005). PCR-based expression analysis and identification of microRNAs. J. RNAi Gene Silencing 1, 44-49.

Macias, S., Plass, M., Stajuda, A., Michlewski, G., Eyras, E., and Caceres, J. F. (2012). DGCR8 HITS-CLIP reveals novel functions for the microprocessor. Nat. Struct. Mol. Biol. 19, 760-766. doi:10.1038/nsmb.2344

Mackowiak, S. D. (2011). Identification of novel and known miRNAs in deepsequencing data with miRDeep2. Curr. Protoc. Bioinformatics 12, 10. doi:10. 1002/0471250953.bi1210s36

Mali, P., Yang, L., Esvelt, K. M., Aach, J., Guell, M., Dicarlo, J. E., et al. (2013). RNA-guided human genome engineering via Cas9. Science 339, 823-826. doi:10.1126/science.1232033

Mapleson, D., Moxon, S., Dalmay, T., and Moulton, V. (2013). MirPlex: a tool for identifying miRNAs in high-throughput sRNA datasets without a genome. J. Exp. Zool. B Mol. Dev. Evol. 320, 47-56. doi:10.1002/jez.b.22483

Mathelier, A., and Carbone, A. (2010). MIReNA: finding microRNAs with high accuracy and no learning at genome scale and from deep sequencing data. Bioinformatics 26, 2226-2234. doi:10.1093/bioinformatics/btq329

Mayoral, J. G., Etebari, K., Hussain, M., Khromykh, A. A., and Asgari, S. (2014). Wolbachia infection modifies the profile, shuttling and structure of microRNAs in a mosquito cell line. PLoS One 9:e96107. doi:10.1371/journal.pone.0096107

Medina, P. P., and Slack, F. J. (2008). microRNAs and cancer: an overview. Cell Cycle 7, 2485-2492. doi:10.4161/cc.7.16.6453

Mencia, A., Modamio-Hoybjor, S., Redshaw, N., Morin, M., Mayo-Merino, F., Olavarrieta, L., et al. (2009). Mutations in the seed region of human miR-96 are responsible for nonsyndromic progressive hearing loss. Nat. Genet. 41, 609-613. doi:10.1038/ng.355

Metpally, R. P., Nasser, S., Malenica, I., Courtright, A., Carlson, E., Ghaffari, L., et al. (2013). Comparison of analysis tools for miRNA high throughput sequencing using nerve crush as a model. Front. Genet. 4:20. doi:10.3389/fgene.2013.00020

Mohorianu, I., Schwach, F., Jing, R., Lopez-Gomollon, S., Moxon, S., Szittya, G., et al. (2011). Profiling of short RNAs during fleshy fruit development reveals stage-specific sRNAome expression patterns. Plant J. 67, 232-246. doi:10.1111/ j.1365-313X.2011.04586.x

Morita, S., Horii, T., Kimura, M., Goto, Y., Ochiya, T., and Hatada, I. (2007). One Argonaute family member, Eif2c2 (Ago2), is essential for development and appears not to be involved in DNA methylation. Genomics 89, 687-696. doi:10.1016/j.ygeno.2007.01.004

Moxon, S., Schwach, F., Dalmay, T., Maclean, D., Studholme, D. J., and Moulton, V. (2008). A toolkit for analysing large-scale plant small RNA datasets. Bioinformatics 24, 2252-2253. doi:10.1093/bioinformatics/btn428

Nam, J. W., Shin, K. R., Han, J., Lee, Y., Kim, V. N., and Zhang, B. T. (2005). Human microRNA prediction through a probabilistic co-learning model of sequence and structure. Nucleic Acids Res. 33, 3570-3581. doi:10.1093/nar/gki668

Ohler, U., Yekta, S., Lim, L. P., Bartel, D. P., and Burge, C. B. (2004). Patterns of flanking sequence conservation and a characteristic upstream motif for microRNA gene identification. RNA 10, 1309-1322. doi:10.1261/rna.5206304

Okamura, K., Hagen, J. W., Duan, H., Tyler, D. M., and Lai, E. C. (2007). The mirtron pathway generates microRNA-class regulatory RNAs in Drosophila. Cell 130, 89-100. doi:10.1016/j.cell.2007.06.028

Okamura, K., Phillips, M. D., Tyler, D. M., Duan, H., Chou, Y. T., and Lai, E. C. (2008). The regulatory activity of microRNA* species has substantial influence on microRNA and 3' UTR evolution. Nat. Struct. Mol. Biol. 15, 354-363. doi:10.1038/nsmb.1409
Pandey, R., Joshi, G., Bhardwaj, A. R., Agarwal, M., and Katiyar-Agarwal, S. (2014). A comprehensive genome-wide study on tissue-specific and abiotic stress-specific miRNAs in Triticum aestivum. PLoS One 9:e95800. doi:10.1371/journal.pone. 0095800

Pantaleo, V., Szittya, G., Moxon, S., Miozzi, L., Moulton, V., Dalmay, T., et al. (2010). Identification of grapevine microRNAs and their targets using high-throughput sequencing and degradome analysis. Plant J. 62, 960-976. doi:10.1111/j.09607412.2010.04208.x

Pantano, L., Estivill, X., and Marti, E. (2011). A non-biased framework for the annotation and classification of the non-miRNA small RNA transcriptome. Bioinformatics 27, 3202-3203. doi:10.1093/bioinformatics/btr527

Pasquinelli, A. E., Reinhart, B. J., Slack, F., Martindale, M. Q., Kuroda, M. I., Maller, B., et al. (2000). Conservation of the sequence and temporal expression of let-7 heterochronic regulatory RNA. Nature 408, 86-89. doi:10.1038/35040556

Pundhir, S., and Gorodkin, J. (2013). microRNA discovery by similarity search to a database of RNA-seq profiles. Front. Genet. 4:133. doi:10.3389/fgene.2013. 00133

Ruby, J. G., Jan, C., Player, C., Axtell, M. J., Lee, W., Nusbaum, C., et al. (2006). Large-scale sequencing reveals 21U-RNAs and additional microRNAs and endogenous siRNAs in C. elegans. Cell 127, 1193-1207. doi:10.1016/j.cell.2006. 10.040

Ruby, J. G., Jan, C. H., and Bartel, D. P. (2007). Intronic microRNA precursors that bypass Drosha processing. Nature 448, 83-86. doi:10.1038/nature05983

Sheng, Y., Engstrom, P. G., and Lenhard, B. (2007). Mammalian microRNA prediction through a support vector machine model of sequence and structure. PLoS One 2:e946. doi:10.1371/journal.pone.0000946

Shi, W., Hendrix, D., Levine, M., and Haley, B. (2009). A distinct class of small RNAs arises from pre-miRNA-proximal regions in a simple chordate. Nat. Struct. Mol. Biol. 16, 183-189. doi:10.1038/nsmb.1536

Smalheiser, N. R., and Torvik, V. I. (2005). Mammalian microRNAs derived from genomic repeats. Trends Genet. 21, 322-326. doi:10.1016/j.tig.2005.04.008

Smith, L. K., Tandon, A., Shah, R. R., Mav, D., Scoltock, A. B., and Cidlowski, J. A. (2013). Deep sequencing identification of novel glucocorticoidresponsive miRNAs in apoptotic primary lymphocytes. PLoS ONE 8:e78316. doi:10.1371/journal.pone.0078316

Sorefan, K., Pais, H., Hall, A. E., Kozomara, A., Griffiths-Jones, S., Moulton, V., et al. (2012). Reducing ligation bias of small RNAs in libraries for next generation sequencing. Silence 3, 4. doi:10.1186/1758-907X-3-4

Stefani, G., and Slack, F. J. (2008). Small non-coding RNAs in animal development. Nat. Rev. Mol. Cell Biol. 9, 219-230. doi:10.1038/nrm2347

Stocks, M. B., Moxon, S., Mapleson, D., Woolfenden, H. C., Mohorianu, I., Folkes, L., et al. (2012). The UEA sRNA workbench: a suite of tools for analysing and visualizing next generation sequencing microRNA and small RNA datasets. Bioinformatics 28, 2059-2061. doi:10.1093/bioinformatics/bts311

Surridge, A. K., Lopez-Gomollon, S., Moxon, S., Maroja, L. S., Rathjen, T., Nadeau, N. J., et al. (2011). Characterisation and expression of microRNAs in developing wings of the neotropical butterfly Heliconius melpomene. BMC Genomics 12:62. doi:10.1186/1471-2164-12-62

Szittya, G., Moxon, S., Santos, D. M., Jing, R., Fevereiro, M. P., Moulton, V., et al. (2008). High-throughput sequencing of Medicago truncatula short RNAs identifies eight new miRNA families. BMC Genomics 9:593. doi:10.1186/1471-21649-593

Taft, R. J., Pang, K. C., Mercer, T. R., Dinger, M., and Mattick, J. S. (2010). Non-coding RNAs: regulators of disease. J. Pathol. 220, 126-139. doi:10.1002/path.2638

Tam, O. H., Aravin, A. A., Stein, P., Girard, A., Murchison, E. P., Cheloufi, S., et al. (2008). Pseudogene-derived small interfering RNAs regulate gene expression in mouse oocytes. Nature 453, 534-538. doi:10.1038/nature06904

Wang, W. C., Lin, F. M., Chang, W. C., Lin, K. Y., Huang, H. D., and Lin, N. S. (2009). miRExpress: analyzing high-throughput sequencing data for profiling microRNA expression. BMC Bioinformatics 10:328. doi:10.1186/14712105-10-328

Wang, X., Zhang, J., Li, F., Gu, J., He, T., Zhang, X., et al. (2005). microRNA identification based on sequence and structure alignment. Bioinformatics 21, 3610-3614. doi:10.1093/bioinformatics/bti562

Wang, Y., Medvid, R., Melton, C., Jaenisch, R., and Blelloch, R. (2007). DGCR8 is essential for microRNA biogenesis and silencing of embryonic stem cell selfrenewal. Nat. Genet. 39, 380-385. doi:10.1038/ng1969

Watanabe, T., Takeda, A., Tsukiyama, T., Mise, K., Okuno, T., Sasaki, H., et al. (2006). Identification and characterization of two novel classes of small RNAs in the 
mouse germline: retrotransposon-derived siRNAs in oocytes and germline small RNAs in testes. Genes Dev. 20, 1732-1743. doi:10.1101/gad.1425706

Watanabe, T., Totoki, Y., Toyoda, A., Kaneda, M., Kuramochi-Miyagawa, S., Obata, Y., et al. (2008). Endogenous siRNAs from naturally formed dsRNAs regulate transcripts in mouse oocytes. Nature 453, 539-543. doi:10.1038/nature06908

Wen, M., Shen, Y., Shi, S., and Tang, T. (2012). miREvo: an integrative microRNA evolutionary analysis platform for next-generation sequencing experiments. BMC Bioinformatics 13:140. doi:10.1186/1471-2105-13-140

Williamson, V., Kim, A., Xie, B., Mcmichael, G. O., Gao, Y., and Vladimirov, V. (2013). Detecting miRNAs in deep-sequencing data: a software performance comparison and evaluation. Brief. Bioinformatics 14, 36-45. doi:10.1093/bib/bbs010

Wu, J., Liu, Q., Wang, X., Zheng, J., Wang, T., You, M., et al. (2013). mirTools 2.0 for non-coding RNA discovery, profiling, and functional annotation based on high-throughput sequencing. RNA Biol. 10, 1087-1092. doi:10.4161/ rna. 25193

Yang, J. H., and Qu, L. H. (2012). DeepBase: annotation and discovery of microRNAs and other noncoding RNAs from deep-sequencing data. Methods Mol. Biol. 822, 233-248. doi:10.1007/978-1-61779-427-8_16

Yang, J. S., Phillips, M. D., Betel, D., Mu, P., Ventura, A., Siepel, A. C., et al. (2011). Widespread regulatory activity of vertebrate microRNA* species. RNA 17, 312-326. doi:10.1261/rna.2537911

Yang, X., and Li, L. (2011). miRDeep-P: a computational tool for analyzing the microRNA transcriptome in plants. Bioinformatics 27, 2614-2615. doi:10.1093/ bioinformatics/btr430

Zeng, Y., and Cullen, B. R. (2003). Sequence requirements for micro RNA processing and function in human cells. RNA 9, 112-123. doi:10.1261/rna.2780503
Zhang, B. H., Pan, X. P., Cox, S. B., Cobb, G. P., and Anderson, T. A. (2006a). Evidence that miRNAs are different from other RNAs. Cell. Mol. Life Sci. 63, 246-254. doi:10.1007/s00018-005-5467-7

Zhang, L., Huang, J., Yang, N., Greshock, J., Megraw, M. S., Giannakakis, A., et al (2006b). microRNAs exhibit high frequency genomic alterations in human cancer. Proc. Natl. Acad. Sci. U.S.A. 103, 9136-9141. doi:10.1073/pnas.0508889103

Zuker, M. (2003). Mfold web server for nucleic acid folding and hybridization prediction. Nucleic Acids Res. 31, 3406-3415. doi:10.1093/nar/gkg595

Conflict of Interest Statement: The authors declare that the research was conducted in the absence of any commercial or financial relationships that could be construed as a potential conflict of interest.

Received: 15 October 2014; accepted: 07 January 2015; published online: 26 January 2015.

Citation: Kang $W$ and Friedländer MR (2015) Computational prediction of miRNA genes from small RNA sequencing data. Front. Bioeng. Biotechnol. 3:7. doi: 10.3389/fbioe.2015.00007

This article was submitted to Bioinformatics and Computational Biology, a section of the journal Frontiers in Bioengineering and Biotechnology.

Copyright (c) 2015 Kang and Friedländer. This is an open-access article distributed under the terms of the Creative Commons Attribution License (CC BY). The use, distribution or reproduction in other forums is permitted, provided the original author(s) or licensor are credited and that the original publication in this journal is cited, in accordance with accepted academic practice. No use, distribution or reproduction is permitted which does not comply with these terms. 